\title{
Comparison of Measurements and Simulation Model of Indoor Signal Level Variation in LTE Networks
}

\author{
Krzysztof Grochla \\ Institute of Theoretical and \\ Applied \\ Informatics of PAS \\ Baltycka 5, Gliwice, Poland \\ kgrochla@iitis.pl
}

\author{
Michał Gorawski \\ Institute of Theoretical and \\ Applied \\ Informatics of PAS \\ Baltycka 5, Gliwice, Poland \\ mgorawski@iitis.pl
}

\author{
Konrad Połys \\ Institute of Theoretical and \\ Applied \\ Informatics of PAS \\ Baltycka 5, Gliwice, Poland \\ kpolys@iitis.pl
}

\begin{abstract}
This paper describes the measurements of the signal level changes in LTE networks in different indoor conditions and compares them with $\mathrm{OMNeT}++$ simulation scenarios. The simulation models are matched with the measurements to have the same average signal level for stationary client. Next the mobility model is applied in the simulation and compared with the measurements of the user moving within a building. The comparison has been executed for signal level changes for mobile phones and tablets in three indoor usage scenarios: stationary, office and home mobility. The results show that the popular mobility and signal propagation models give a significantly different variance of the changes of signal level compared with the measurements and are not correctly representing the variance caused by indoor fading.
\end{abstract}

\section{Categories and Subject Descriptors}

C.2.1 [Computer-Communication Networks]: Network Architecture and Design-Wireless communication

\section{General Terms \\ Theory}

\section{Keywords}

wireless, indoor, signal propagation, simulation, measurements

\section{INTRODUCTION}

Communication by mobile networks became part of our living. Among various technologies used for providing indoor and outdoor coverage the LTE (Long Term Evolution) is gaining most attention and is becoming a natural upgrade for the GSM and UMTS networks. The goal of LTE was to increase the capacity and speed of wireless data networks. The LTE provides data transmission peek rates of $300 \mathrm{MBit} / \mathrm{s}$. Both indoor and outdoor and it has been widely deployed around the world.
The wireless networks for mobile devices consist of multiple base stations and provide coverage over a large area. The structure of the network may be quite complex, with different size of the cells [1]. As the mobile clients move, the communication between them and the network is handled by different eNodeBs (base stations). The wireless transmission is heavily influenced by the radio signal propagation conditions. The received signal level changes in time due to node mobility and due to changes in the environment and fading. This is the case especially for indoor usage of LTE devices, when even a slight change in the device location may result in large change of measured received signal level and the radio channel fading is significant.

\subsection{Motivation}

The discrete event simulations are widely used for the evaluation of the wireless network protocols and algorithms. The simulation models rely on two elements to proper represent the transmission conditions and attenuation of the radio link in mobile networks: the radio signal propagation model and the user mobility model. In this paper we evaluate commonly used mobility and signal propagation models with measurements of the signal level for indoor LTE user. We measure how the LTE received signal level changes in time for LTE User Equipment (UE) when it is mobile or stationary in indoor location, and compare to the simulation results. In simulation we try to reproduce similar transmission conditions and apply a commonly used mobility model, to show how the measurements and simulation results differ.

\subsection{Signal level variation and mobility}

A large number of models representing the radio signal propagation have been proposed in the literature. These models usually calculate the path loss which can be defined as the ratio of the transmitted power to received power, usually expressed in decibel. Path loss models can be divided in two groups - empirical and statistical models. Most common and basic signal propagation model is FreeSpace which assumes that electromagnetic wave goes through space without obstacles and any reflections or diffraction. Another popular model is the Okumura model which is widely used to calculate propagation in urban areas. It is simple and provides good accuracy for early cellular systems. It is commonly set as a base for many other models. It can be used for frequencies up to $3 \mathrm{GHz}$ with distance between transmitter and receiver around $100 \mathrm{~km}$. Next one, the Hata model, also known as the Okumura-Hata, is an empirical formulation of 
graphical data from the Okumura model. It defines three types of terrain - urban, suburban and open areas. The COST-231 Walfisch-Ikegami model is a combination of $\mathrm{J}$. Walfisch and F. Ikegami models. This model gives high accuracy in urban environments because it considers only the buildings in the vertical plane between the transmitter and the receiver. The Stanford University Interim (SUI) model uses similar approach with three types of terrain defined, but is more accurate for frequencies near to $2 \mathrm{GHz}[2,7,8$, 9]. The user mobility is the key factor that determines the changes of signal level, as it depends on the user location and distance from the base station. A large number of mobility models can be found in the literature, from very simple, such as Random Waypoint (RW) [6] or Gauss-Markov (GM) [6], to models capturing human behavior properties like Lévy flight. Most of the mobility models however provides data about outdoor mobility or assumes that there are no buildings on the modeled area. The indoor mobility has been studied more recently and few models are proposed to simulate movement inside buildings. The rest of the paper is organized as follows: Section 2 describes simulation model, Section 3 gives detailed description of our signal level measurements and the simulation results. Section 4 concludes the paper.

\section{SIMULATION MODEL}

We tried to represent similar conditions in the widely used OMNeT ++ environment [10]. The simulation assumes two scenarios, representing respectively home and office location. The first one consists of area with dimensions $400 \times 400 \mathrm{~m}$, one base station and an obstacle which simulate the office with mobile node inside. The obstacle has dimensions of 9 x $25 \mathrm{~m}$. The second case is similar but it represents user at home with simulation area of $80 \times 80 \mathrm{~m}$ and obstacle with wall indoor. Dimensions and arrangement of all elements are based on Google Maps satellite view and database with locations of Base Transceiver Stations. We assume that the nearest base station is used. The attenuation of wall and the attenuation per meter was set to, respectively, $10 \mathrm{~dB}$ and $0.8 \mathrm{~dB}[5]$. In simulations we represented the same distances and similar walls as in the locations for which the measurements were carried on. We test UE mobility by applying two very commonly used mobility models: the Random Waypoint (RW) and Gauss-Markov (GM) mobility models in each experiment. Figures from 1a to $3 b$ show case with BS power set to $40 \mathrm{dBm}$. Simulation results shown in figures $4 \mathrm{a}-6 \mathrm{~b}$ represents experiments with BS power set to value which allow us to achieve the same signal level value for stationary UE as the average signal level in measurements. To calculate the radio signal propagation we use the SUI propagation model, described in more details in $[2,3]$.

\section{SIGNAL LEVEL MEASUREMENTS AND SIMULATION RESULTS}

We aimed in comparison of the distribution of signal level in simulations with the measured values [4]. The comparison was used for two TX power setting: a typical value of $40 \mathrm{dBm}$ and values matched to achieve the same average signal level as from real measurements. The results of the comparison for the home mobility scenario with TX power set to $40 \mathrm{dBm}$ are presented on Fig.1a (GM mobility) and Fig.1b (RW mobility). The measurements show two dis-

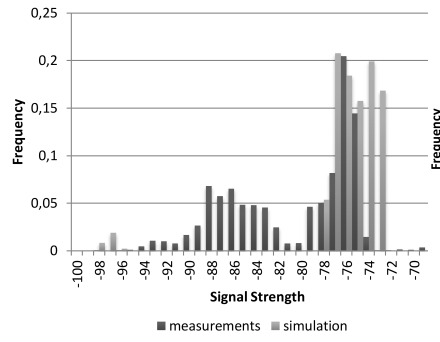

(a) Gauss-Markov $40 \mathrm{dBm}$

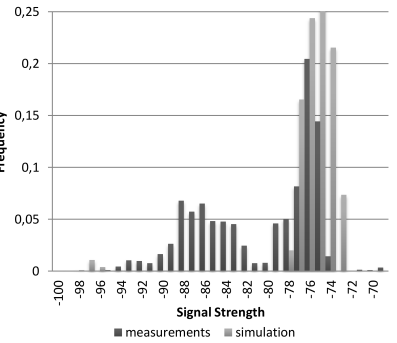

(b) RandomWaypoint $40 \mathrm{dBm}$
Figure 1: Comparison of signal strength changes between measured and simulated values in home environment

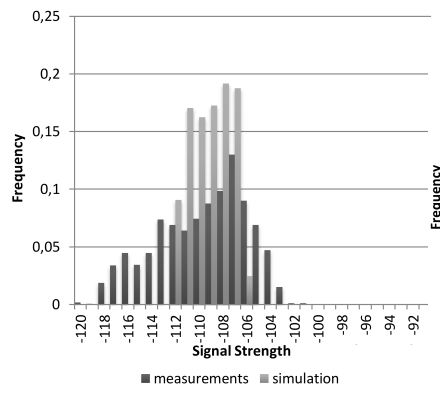

(a) Gauss-Markov $40 \mathrm{dBm}$

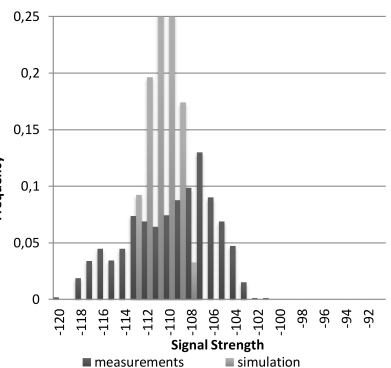

(b) RandomWaypoint $40 \mathrm{dBm}$
Figure 2: Comparison of signal strength changes between measured and simulated values in office environment

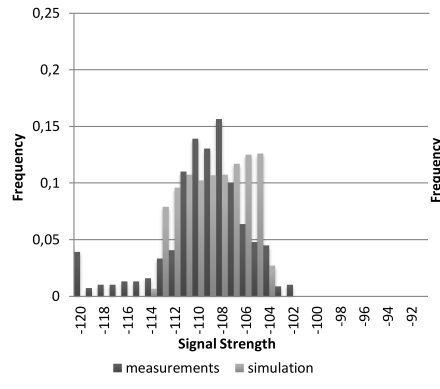

(a) Gauss-Markov $40 \mathrm{dBm}$

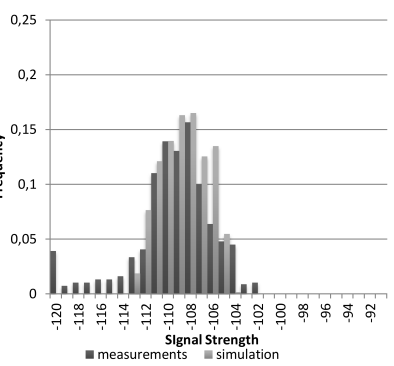

(b) RandomWaypoint $40 \mathrm{dBm}$
Figure 3: Comparison of signal strength changes between measured and simulated values of a moving office worker

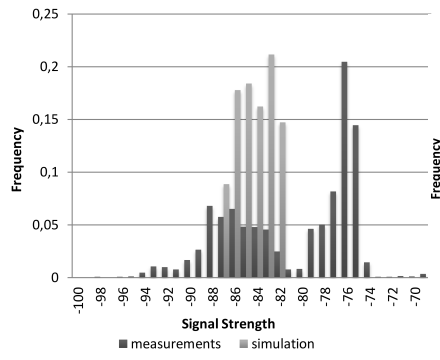

(a) Gauss-Markov

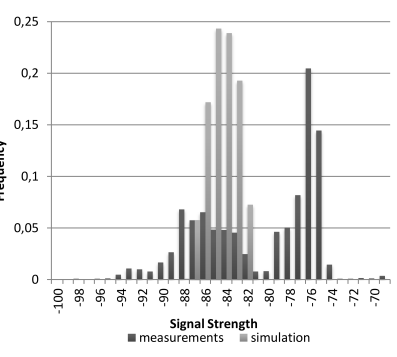

(b) RandomWaypoint
Figure 4: Comparison of signal strength changes between measured and simulated values in home environment

tinguishing maximums, one around values $-79 \mathrm{dBm}$ to -74 $\mathrm{dBm}$ and the second one $-94 \mathrm{dBm}$ to $-82 \mathrm{dBm}$. This is most 


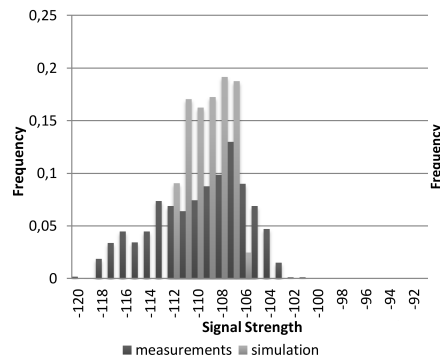

(a) Gauss-Markov

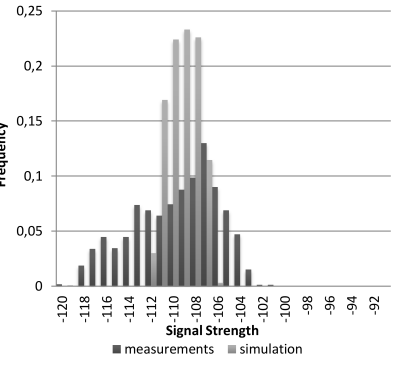

(b) RandomWaypoint
Figure 5: Comparison of signal strength changes between measured and simulated values in office environment

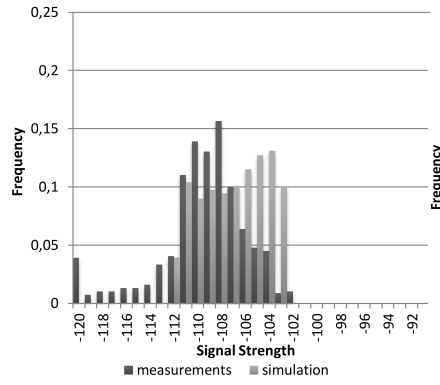

(a) Gauss-Markov

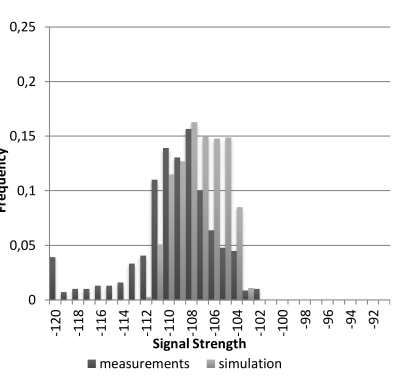

(b) RandomWaypoint
Figure 6: Comparison of signal strength changes between measured and simulated values of a moving office worker

probably caused by the wall partially separating the room where measurement took place. We simulated this case in the model and although we obtained similar effect of signal level concentrating in two different levels, the results of the simulation are considerably different than the measurements. The simulation showed much larger dispersion of the signal levels. The office scenario with TX power set to 40 $\mathrm{dBm}$ is presented in Fig.2a (GM mobility) and Fig.2b (RW mobility). Histogram shows the total measurements taken from 10 different locations inside office space, in the course of 10 working days. The simulation of office suite, included limiting the whole office space from $9 \times 25 \mathrm{~m}$ to $5 \times 10 \mathrm{~m}$. The simulated results does not match original measurement values. The office worker movement in office space (Figures 3a (GM mobility) and 3b (RW mobility)) was simulated on the whole $9 \times 25 \mathrm{~m}$ simulation space. The simulation results for Gauss-Markov movement differ from the original measurements, for the Random Waypoint movement we can observe some similarity, but with considerable cut-offs from both sides, comparing to original measurements. Next series of simulations is done with variable Base Station power, set adequately to match the simulated value of signal change with the average value of signal change in a real life measurements. For home environment Fig. 4a (GM mobility) and Fig. 4b (RW mobility) shows considerable difference of simulated results and measurements. For the office suite (Fig. 5a (GM mobility) and Fig. 5b (RW mobility)), and office mobility (Fig. 6a (GM mobility) and Fig. 6b (RW mobility)) we did not obtain simulated results similar to real-life measurements. When we compared the simulation to the measurement gathered for moving office worker (Fig. $6 \mathrm{a}$ and $6 \mathrm{~b}$ ) the measurement were showing much more probability mass in the lower signal level area. This suggest that the simulation is not representing some interferences which decrease the signal level over short periods of time.

\section{CONCLUSIONS}

The simulations presented in following paper tried to reproduce the characteristics of a signal change in typical environment (at home, in office suite, at work movement). Our experiments showed that there is a significant difference between the signal level variance observed during measurements and the variance produced by a simulation model. Even a stationary UE is subjected to significant signal change in time. Most of the radio signal propagation models used in discrete event simulators, like $\mathrm{OMNeT}++$ or NS-3 do not model this. The distributions of measured signal level changes in LTE show greater variance than achieved during simulation and the correct tuning of the simulation model is virtually impossible. When the model is parametrized to reproduce the measurements of a device in one location (RW model in Fig.3b), the same model in other location provides results which are significantly different from measured values (RW model in Fig.2b). [4]

\section{ACKNOWLEDGMENTS}

This work was funded by the Polish National Centre for Research and Development, under research grant no. LIDER/10/194/L-3/11/, http://zosb.iitis.pl.

\section{REFERENCES}

[1] A. Domański, J. Domańska, and T. Czachórski. The impact of self-similarity on traffic shaping in wireless lan. In Next Generation Teletraffic and Wired/Wireless Advanced Networking. Springer, 2008.

[2] V. Erceg and et al. An empirically based path loss model for wireless channels in suburban environments. Selected Areas in Communications, IEEE, 1999.

[3] G. Giambene and T. A. Yahiya. Lte planning for soft frequency reuse. In Wireless Days, IFIP. IEEE, 2013.

[4] M. Gorawski, K. Grochla, and K. Polys. Variability of lte signal in indoor environment. In Internet in the Information Society, X Scientific Conference, 2015.

[5] D. M. Rose, T. Jansen, T. Werthmann, U. Türke, and T. Kürner. The ic 1004 urban hannover scenario-3d pathloss predictions and realistic traffic and mobility patterns. 2013.

[6] R. R. Roy. Handbook of Mobile Ad Hoc Networks for Mobility Models. Springer, 2010.

[7] T. K. Sarkar, Z. Ji, K. Kim, A. Medouri, and M. Salazar-Palma. A survey of various propagation models for mobile communication. Antennas and Propagation Magazine, IEEE, 2003.

[8] N. Shabbir, M. T. Sadiq, H. Kashif, and R. Ullah. Comparison of radio propagation models for long term evolution (lte) network. arXiv preprint arXiv:1110.1519, 2011.

[9] N. Shebani, A. Mohammed, M. Mosbah, and Y. Hassan. Simulation and analysis of path loss models for wimax communication system. In 3th ICDIPC. The Society of Digital Information and Wireless Communication, 2013.

[10] A. Varga et al. The omnet++ discrete event simulation system. In Proceedings of the European Simulation Multiconference, volume 9. sn, 2001. 